\title{
OXYPETALUM RADINSII (APOCYNACEAE: ASCLEPIADOIDEAE), UNA NUEVA ESPECIE ENDÉMICA DE MISIONES, ARGENTINA
}

\author{
HÉCTOR A. KELLER ${ }^{1}$
}

\begin{abstract}
Summary: Keller, H. A. 2015. Oxypetalum radinsii (Apocynaceae: Asclepiadoideae), a new species endemic to Misiones Province, Argentina. Bonplandia 24(1): 51-56.

Based on specimens collected in the Misiones Province, Argentina, a new species of the genus Oxypetalum (Apocynaceae: Asclepiadoideae) related to $O$. argentinum, $O$. humile and $O$. microphyllum is described here. Currently known populations occur in fragments of grasslands surrounded by subtropical forest.
\end{abstract}

Key words: Argentinean flora, rock outcrops grasslands, species critically endangered, taxonomy.

Resumen: Keller, H. A. 2015. Oxypetalum radinsii (Apocynaceae: Asclepiadoideae), una nueva especie endémica de Misiones, Argentina. Bonplandia 24(1): 51-56.

Sobre la base de ejemplares recolectados en Misiones, Argentina, se describe una nueva especie del género Oxypetalum (Apocynaceae: Asclepiadoideae) afín a $O$. argentinum, $O$. humile y $\mathrm{O}$. microphyllum. Las poblaciones hasta ahora conocidas se localizan en fragmentos de pastizales rodeados de selva subtropical.

Palabras clave: Campos rupestres, especie en peligro crítico, flora argentina, taxonomía.

\section{Introducción}

En su esquema fitogeográfico de la provincia de Misiones Raúl N. Martínez Crovetto (1963) describe el distrito del Urunday como una subdivisión del Sector Misionero, caracterizada por la presencia de poblaciones de Astronium balansae Engl. (Anacardiaceae) sobre lomadas pedregosas. De acuerdo con el autor, el límite de este distrito con el distrito de los Laureles no es perfectamente regular, sino que se verifica en forma de engranaje muy irregular y dejando enclaves o islas a uno y otro lado (p. 188). Entre estos enclaves del distrito del Urunday se destacan una serie de pastizales insularizados situados en el Depto. Cainguás, en pleno corazón de la selva Misionera.

Recientes expediciones botánicas que interceptaron algunos de estos campos naturales rodeados de selva primaria posibilitaron la recolección de material de herbario de una especie del género Oxypetalum R. Br. (Apocynaceae: Asclepiadoideae) aún no descripta por la ciencia. Este género cuenta con alrededor de 120 especies nativas de regiones tropicales, subtropicales y templadas de América; distribuidas desde el centro de la Argentina hasta México (Farinaccio, 2006). De las 41 especies del género citadas para la Argentina, 25 han sido documentadas para la

\footnotetext{
${ }^{1}$ Instituto de Botánica del Nordeste, CC 209, 3400-Corrientes, Argentina. Investigador CONICET. Facultad de Ciencias Forestales, Universidad Nacional de Misiones - E-mail: hakeller2000@yahoo.com.ar
} 
provincia de Misiones, 13 de las cuales son plantas trepadoras y 12 sufrútices erectos o rastreros (Farinaccio \& Keller, 2014), como el taxón que se describe aquí es un sufrútice erecto, la presente contribución permite igualar esta proporción.

\section{Material y Métodos}

Las campañas de recolección fueron realizadas en diciembre de 2014 y marzo de 2015. Mediante el uso de un geoposicionador satelital se tomaron puntos para determinar la distribución de las poblaciones halladas. El material recolectado en campo fue procesado y depositado en los herbarios CTES, SI y CGMS. Se estudiaron caracteres vegetativos y reproductivos de material fresco y disecado, de la especie nueva y de especies afines. Una cámara fotográfica con alta resolución y un microscopio estereoscópico fueron utilizados para obtener imágenes empleadas en el proceso de estudio del material y para confeccionar las ilustraciones.

\section{Resultados}

\section{Oxypetalum radinsii $\mathrm{H}$. A. Keller, sp.nov.}

Figs. 1 y 2

The new species shares some morphological characters with $O$. argentinum Malme, $O$. humile (Morong) Hassl. and O. microphyllum Hook. \& Arn.; these species are small plants, the internodes, leaf blades, flowers and fruits have similar shape and dimensions and all of them possess umbelliform inflorescences with few flowers. However, in O. radinsii the apical anthers membrane is wider than longer and the corona lobes are 1-1.2 $\mathrm{mm}$ long, shorter than in the related species.

Typus: ARGENTINA. Misiones. Cainguás: Cuñá Pirú. 2706'14,1”S, 5459'8,7”W, $224 \mathrm{~m}$, 04-III-2015, fl, fr, H. A. Keller, J. J. Araujo \& M. M. Franco 12770 (holotypus CTES!, isotypus CGMS!).

Sufrútice latescente erecto, ramificado en la base, ramas fértiles $30-60 \mathrm{~cm}$ alt.; tallos cilíndricos, pilosos, pelos multicelulares, uniseriados, ca. $1 \mathrm{~mm}$ long; entrenudos 0,8 $2,5 \mathrm{~cm}$ long. Hojas opuestas, subsésiles, las superiores erguidas y revolutas; pecíolos 0,2-1 mm long.; láminas ovadas a ovadolanceoladas, con frecuencia falcadas, revolutas, (2-) 4-15 × 2-9 mm, concoloras, pilosas sobre la vena media y las secundarias; margen entero, ciliado, base redondeada, ápice agudo, venación broquidódroma. Inflorescencias extra-axilares umbeliformes, erectas, alternas, con 1-3 flores; pedúnculos 3-7,5 mm long, pilosos; brácteas filiformes, pilosas 2-2,5 $\times$ 0,1-0,2 mm; pedicelos 5-11 mm long., pilosos. Cáliz dividido hasta cerca de la base, verde-amarillento; lóbulos estrechamente lanceolados, agudos, 2-2,5 × 0,7-1 mm, acuminados, pilosos en la base, glabros en el ápice, 4-6 coléteres en el seno. Corola rotácea, lóbulos lanceolados 5,6-6,5 × 1,8-2 mm, rojizos a verde-amarillentos, retorcidos en el tercio distal, basalmente pilósulos, glabros en el ápice. Corona blanca, carnosa, lóbulos $1-1,2 \times 0,8-1 \mathrm{~mm}$, escindidos casi hasta la base conformando cada uno de ellos dos piezas tortuosas con forma de "s". Anteras rectangulares, ca. $1 \times 0,6 \mathrm{~mm}$, membrana apical rectangular, 0,4-0,5 × 0,5-0,6 mm. Retináculo oblongo, contraído en la parte distal, 0,26$0,33 \times 0,09-0,1 \mathrm{~mm}$, caudículas $0,12-0,2 \mathrm{~mm}$ long., aplanadas, translúcidas, sin dientes, polinias elípticas, $0,52-0,61 \times 0,20-0,22 \mathrm{~mm}$. Ginostegio blanco con tonos rosados, 1,8-2 $\mathrm{mm}$ long, brevemente estipitado, apéndice estigmático ca. $0,5 \mathrm{~mm}$ long., blanco, exerto, bífido, ramas cónicas divergentes o incurvas. Folículos generalmente simples, erguidos, rectos o algo incurvos, 3-4 cm long. $\times 0,4-0,5$ $\mathrm{cm}$ diám., superficie externa amarillenta, con escasos pelos retrorsos; placenta filiforme, marrón claro. Semillas 7-13 por folículo, pardas, ovadas, 3-6 × 2-3 mm, superficie con crestas y mamilas, margen dentado, penacho de 1,2-2 cm long.

Etimología: Especie dedicada al profesor José Antonio Radins del Ministerio de Ecología Recursos Naturales Renovables y Turismo, Gobierno de la Provincia de Misiones, entusiasta defensor y promotor de la flora de Misiones, quien me dio las primeras 


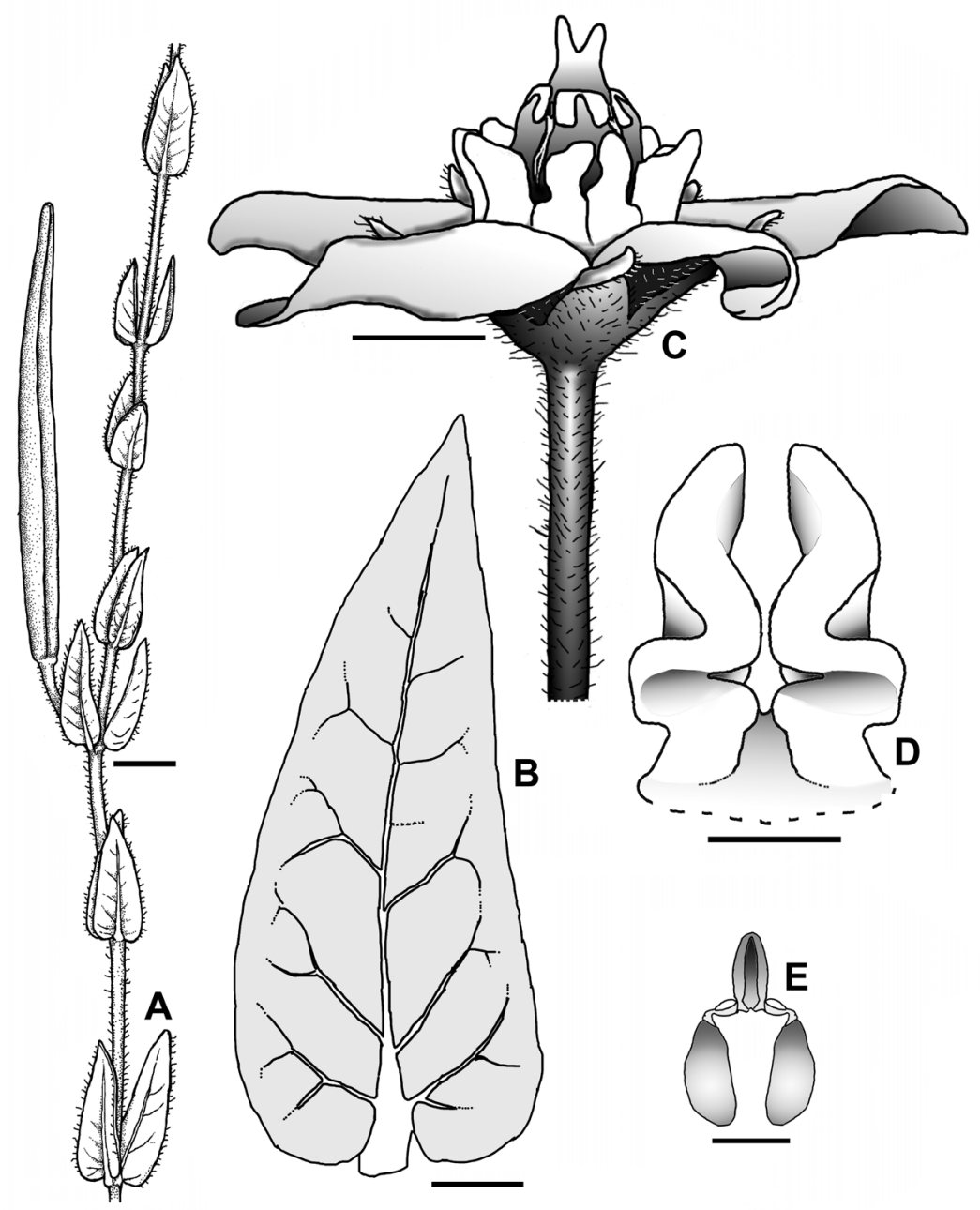

Fig. 1. Oxypetalum radinsii H. A. Keller. A: Segmento de rama con fruto cerrado. B: Contorno de la hoja mostrando venación. C: Flor. D: Corona en vista ventral. E: Polinario. (A-E: holotypus). Escalas en mm. A: 6; B: 3; C: 2; D: 0,6 y E: 0,3. Diseño del autor.

referencias sobre la localidad tipo, donde fotografiaron ejemplares del taxón que aquí se describe.

Distribución y hábitat: La especie es endémica de un sector de la alta cuenca del arroyo Cuñá Pirú, Dep. Cainguás, Misiones, Argentina. En este sector se encuentran alrededor de una veintena de pastizales insularizados en medio de la selva, en tres de los cuales se hallaron poblaciones de $O$. radinsii.

En estos ambientes singulares se destacan algunos componentes leñosos arbóreos y arbustivos como Astronium balansae Engl. (Anacardiaceae), Anadenanthera colubrina
(Vell.) Brenan (Fabaceae) y Aloysia gratissima (Gillies \& Hook. ex Hook.) Tronc. (Verbenaceae) que demarcan el ecotono con la selva y con frecuencia regeneran hacia el interior del pastizal. Sobre estos especímenes leñosos suelen encaramarse algunas plantas trepadoras tales como Dioscorea campestris Griseb., D. multiflora Mart. ex Griseb. (Dioscoreaceae), Ditassa burchellii Hook. \& Arn., Oxypetalum appendiculatum Mart. (Apocynaceae), entre otras. En los sitios desprovistos de dosel leñoso, diversas especies subarbustivas y herbáceas medran entre las matas graminosas y las rosetas de bromeliáceas; además de Oxypetalum radinsii (Apocynaceae) 

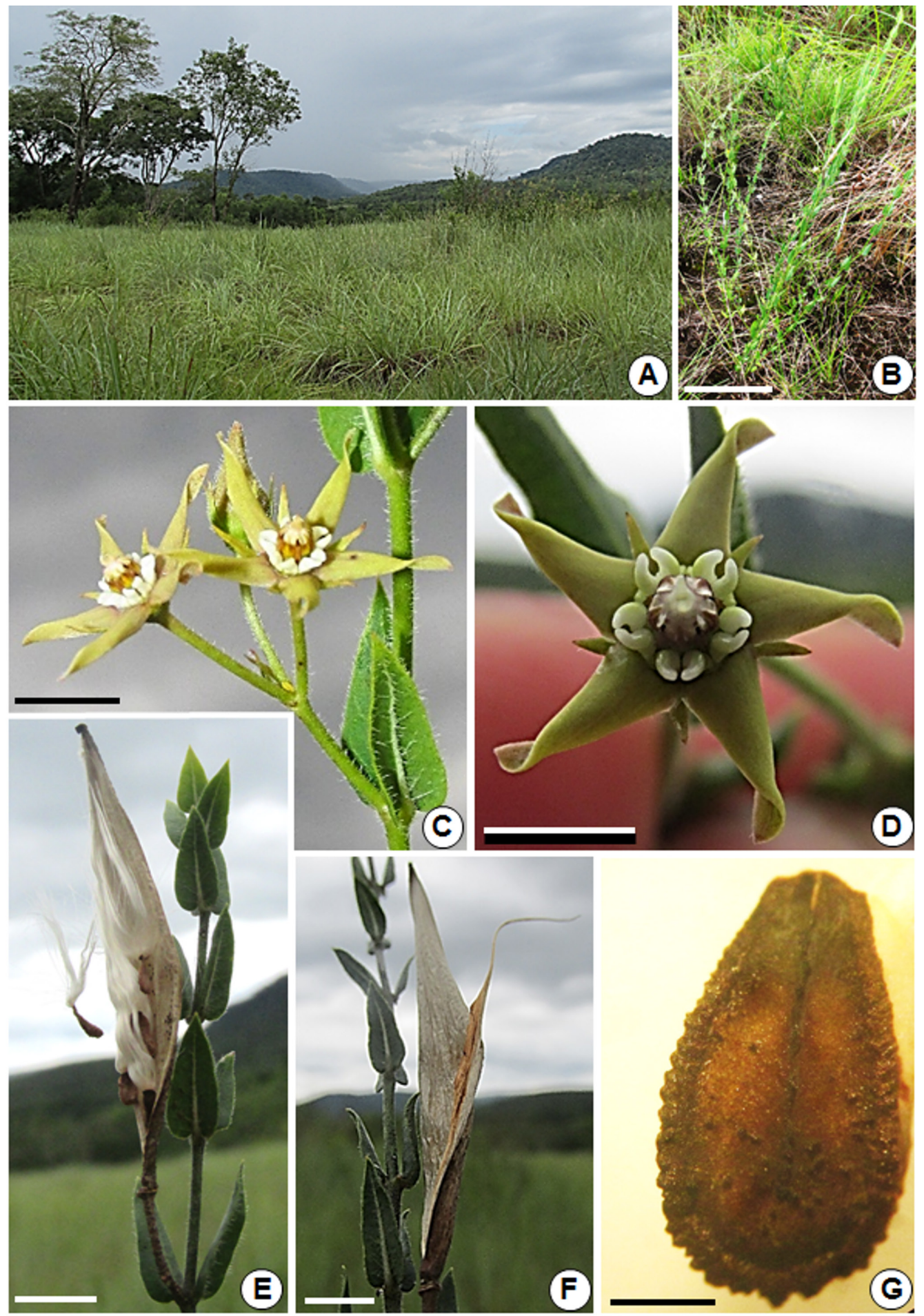

Fig. 2. Oxypetalum radinsii H. A. Keller. A: Ambiente. B: Planta. C: Inflorescencia. D: Flor en vista superior. E: Fruto con semillas. F: Fruto exhibiendo la placenta. G: Semilla. (A-E: Keller et al.12763, CTES). Escalas en cm. B: 10; C: 0,6; D: 0,5; E: 0,8; F: 0,6 y G: 0,12. 
cabe mencionar entre estas a Cuphea linarioides Cham. \& Schltdl. (Lythraceae), Lippia sclerophylla Briq. (Verbenaceae), Skeptrostachys paraguayensis (Rchb. f.) Garay. (Orchidaceae). Por su parte, Cypella suffusa Ravenna (Iridaceae) es también una especie endémica de estos pastizales insularizados (L. Deble com. pers.).

Fenología y observaciones ecológicas: La especie florece desde diciembre hasta marzo y fructifica desde enero hasta abril. Se han observado algunos melipónidos y lepidópteros que visitan sus flores. El hallazgo de un segmento de probóscide de lepidóptero atascado en el retináculo de un polinario estudiado sugiere que, como lo indica Schulz
(1937) para otros representantes de este grupo de plantas, algunos visitantes son capturados por las flores que frecuentan durante el proceso de polinización.

Sólo se conocen hasta el presente cuatro poblaciones situadas en la localidad típica. Estas poblaciones cuentan con pocos individuos. Ello sugiere que la especie debe considerarse en peligro crítico si se siguen los lineamientos establecidos por IUCN (2001).

Paratypi. ARGENTINA. Misiones: Dep. Cainguás, Cuñá Pirú, 2706'54,1"S $54^{\circ} 58^{\prime} 26,3^{\prime \prime} \mathrm{W}, 210 \mathrm{~m}, 28$-XII-2014, H. A. Keller \& N. G. Keller 12581 (CTES); ídem, 2706'13,1"S 5459'20,3”'W, 220 m, 04-III-2015, H. A. Keller et al. 12763 (CTES, SI).

\section{Clave para diferenciar Oxypetalum radinsii de las especies afines}

1. Plantas rastreras; lóbulos del cáliz de hasta 1,8 mm long.; lóbulos de la corola de hasta $3 \mathrm{~mm}$ long. ........ O. microphyllum

1'. Plantas erectas, lóbulos del cáliz nunca menores a $2 \mathrm{~mm}$ long.; lóbulos de la corola de más de $4 \mathrm{~mm}$ long.

2. Cabeza del ginostegio oculta en el interior de la corona. Lóbulos de la corona 1,5-2 mm long. Membrana apical de las anteras más larga que ancha a casi isodiamétrica.

3. Lóbulos de la corola $6 \times 4 \mathrm{~mm}$. Apéndice estigmático bífido ca. $4 \mathrm{~mm}$ long., sobrepasando el nivel de la corona.

O. argentinum

3'. Lóbulos de la corola 4,5 × 1,2 mm. Apéndice estigmático breve y crasso, nunca sobrepasando el nivel de la corona.

O. humile

2'. Cabeza del ginostegio exerta. Lóbulos de la corona de 1-1,2 mm long. Membrana apical de las anteras notablemente más ancha que larga, de 0,4-0,5 × 0,5-0,6 mm. O. radinsii

\section{Discusión}

Además de los caracteres explicitados en la diagnosis y en la clave precedente, $O$. radinsii y las especies afines también pueden diferenciarse entre sí considerando solamente la morfología y dimensiones del apéndice estigmático. Éste es breve y craso en $O$. humile, mientras que en las otras tres especies es visiblemente bífido y alargado, de $4 \mathrm{~mm}$ long en $O$. argentinum y de $0,5 \mathrm{~mm}$ en $O$. microphyllum y $O$. radinsii. El apéndice estigmático de la especie que aquí se describe se bifurca en dos ramas cónicas, divergentes o incurvas, mientras que en $O$. microphyllum se divide en dos ramas filiformes convergentes.

Si se toma en consideración las dimensiones de las piezas del perianto, $O$. radinsii guarda mayor afinidad con $O$. argentinum, presentando ambas especies los lóbulos del cáliz y la corola más anchos y largos que en las otras dos especies. En O. microphyllum los lóbulos del cáliz miden $1,8 \times 0,5 \mathrm{~mm}$ y los de la corola $3 \times 1 \mathrm{~mm}$ y en $O$. humile miden $2 \times 0,5 \mathrm{~mm}$ y $4,5 \times 1,2 \mathrm{~mm}$ respectivamente. Por su parte, en $O$. argentinum los lóbulos del cáliz miden 3,5 × 0,8-0,9 mm y los de la corola $6 \times 2 \mathrm{~mm}$ (Meyer, 1943; Meyer \& Bacigalupo, 1979), aproximándose más a las dimensiones presentadas aquí en la descripción de $O$. radinsii. Lo mismo ocurre con el polinario, $O$. argentinum y $O$. radinsii presentan polinias de más de 0,4 mm long., mientras que en las otras dos especies estas apenas alcanzan los 0,3 $\mathrm{mm}$ long. 


\section{Agradecimientos}

Deseo expresar mi gratitud al profesor José Antonio Radins por la información brindada. A Jorge J. Araujo, Marcelo M. Franco, Néstor G. Keller, Nancy G. Paredes y Rafael Keller por su colaboración durante las campañas de recolección.

\section{Bibliografía}

FARINACCIO, M. A. 2006. Sistemática molecular de Oxypetalum R. Br. (Apocynaceae, Asclepiadoideae). Tese de Doutorado. Universidade de São Paulo, São Paulo. 142 pp. + anexos.
FARINACCIO, M. A. \& H. A. KELLER. 2014. Novelties in Oxypetalum (Apocynaceae-Asclepiadoideae) for the Argentine Flora. Phytotaxa 184: 109-114.

IUCN. 2001. The IUCN red list of threatened species, version 2001.4. IUCN Red List Unit, Cambridge U.K. Available from: http:/www.iucnredlist.org/ (accessed 11 March 2014).

MARTÍNEZ-CROVETTO, R. 1963. Esquema fitogeográfico de la provincia de Misiones (República Argentina). Bonplandia 1: 171-224.

MEYER, T. 1943. Revisión de las especies argentinas del género Oxypetalum (Asclepiadaceae). Lilloa 9: 5-72.

MEYER, T. \& N. M. BACIGALUPO. 1979. Asclepiadaceae. En A. Burkart (ed.), Fl. Il. Entre Ríos, Colecc. Ci. Inst. Nac. Tecnol. Agropecu. 6(5a): 103-147.

SCHULZ, A. G. 1937. Las asclepiadáceas del territorio del Chaco. Lilloa 1: 347-391.

Original recibido el 18 de marzo de 2015; aceptado el 17 de abril de 2015. 